\title{
p53 codon 72 polymorphism and human papillomavirus associated skin cancer
}

\author{
D P O’Connor, E W Kay, M Leader, G J Atkins, G M Murphy, M J E M F Mabruk
}

Pathology

Department, Royal

College of Surgeons in

Ireland, $123 \mathrm{St}$

Stephen's Green,

Dublin 2, Ireland

D P O'Connor

E W Kay

$M$ Leader

M J E M F Mabruk

Department of Microbiology, Moyne

Institute of Preventive

Medicine, Trinity

College, Dublin 2,

Ireland

G J Atkins

\section{Dermatology \\ Department, \\ Beaumont Hospital, \\ Beaumont, Dublin 9, \\ Ireland \\ G M Murphy \\ Correspondence to: Dr Mabruk, Pathology Department, Beaumont Hospital, Beaumont Road, Dublin 9, Ireland mabruk@indigo.ie \\ Accepted for publication 9 November 2000}

\begin{abstract}
BackgroundlAims-Non-melanoma skin cancers frequently harbour multiple human papillomavirus (HPV) types. A recent report suggests that a polymorphism of the p53 tumour suppressor gene that results in the substitution of a proline residue with an arginine residue at position 72 of the p53 protein might act as a risk factor in HPV associated malignancies. This study aimed to determine the following: (1) the relation between HPV infection and the development of cutaneous squamous cell carcinoma (SCC), and (2) whether there is a correlation between p53 codon 72 polymorphism and the development of SCC.

Methods-Blood samples were taken from 55 patients with skin cancer (both renal transplant recipients and immunocompetent patients with skin cancer) and 115 ethnically matched volunteers. A polymerase chain reaction based assay was used to determine p53 codon 72 genotypes. In addition, 49 benign and malignant lesions from 34 of the patients with skin cancer and 20 normal human skin samples from 20 of the control volunteers were examined for HPV.
\end{abstract}

Results-The proportions of p53 codon 72 genotypes found were $78 \%$ arginine homozygous, $2 \%$ proline homozygous, and $20 \%$ heterozygous among patients with skin cancer and $79 \%$ arginine homozygous, $3.5 \%$ proline homozygous, and $17.5 \%$ heterozygous among the control population. Statistical analysis showed no significant differences in the distribution of the two p53 isoforms between the patients with skin cancer and the control population. The predominant viral types detected in both the patients and the control group were EV associated HPVs, although the incidence was lower in normal skin samples than in malignant lesions or viral warts.

Conclusions-These results suggest that in a Celtic population there is no correlation between the presence of HPV, the p53 codon 72 arginine polymorphism, and the development of skin cancer. (F Clin Pathol 2001;54:539-542)

Keywords: p53 codon 72 polymorphism; human papillomavirus; skin cancer

Non-melanoma skin cancers (NMSCs) are the most frequent cancers in white populations. NMSCs often harbour multiple human papillomaviruses (HPVs), although a causal role for the virus in cutaneous tumorigenesis is yet to be confirmed. A recent report ${ }^{1}$ suggests that a common polymorphism at codon 72 of the p53 tumour suppressor gene ${ }^{2}$ might be a risk factor in the development of HPV associated cancers. The replacement of a proline residue with an arginine residue at position 72 of the p53 gene product marginally increased the susceptibility of the protein to degradation by the E6 oncoprotein of high risk HPV types 16 (HPV16) and 18. The presence of an arginine residue was also found to increase greatly the susceptibility of p53 to degradation by the E6 protein of low risk HPV type 11 .

Subsequent reports ${ }^{3}$ demonstrated that $^{4}$ individuals who were homozygous for the arginine variant of the protein were at no greater risk of developing cervical cancer. However, one report suggests that in Swedish and Italian women with HPV-16 positive cervical disease, the incidence of cancer was higher in those patients homozygous for the arginine isoform. ${ }^{5}$

Because cutaneous malignancies often harbour multiple low risk HPV types, ${ }^{6-8}$ and the arginine isoform of p53 is susceptible to low risk HPV E6 mediated degradation, individuals with the arginine variant of p53 might have an increased risk of developing skin cancer.

Here, we examine the possible role of this polymorphism in the development of skin cancer. The distribution of p53 codon 72 alleles was determined in 55 patients with skin cancer (both renal transplant recipients and immunocompetent patients with skin cancer) and in 115 ethnically matched control volunteers.

The HPV status of 34 of the patients with skin cancer and 20 of the control volunteers was also examined to determine the prevailing viral types in each group.

\section{Methods}

PATIENTS AND VOLUNTEERS

Peripheral blood for the p53 codon 72 polymorphism analysis was taken from 55 patients with skin cancer attending the dermatology clinic at Beaumont Hospital and from 115 matched ethnic Irish control volunteers. Forty nine benign and malignant skin lesions comprising viral warts (19), squamous cell carcinomas (SCCs; 21), and normal skin (nine) from 34 of the 55 patients with skin cancer were examined to determine the HPV types present. Twenty normal skin samples from 20 of the control volunteers were also examined for HPV. We obtained ethics committee approval for our study. 


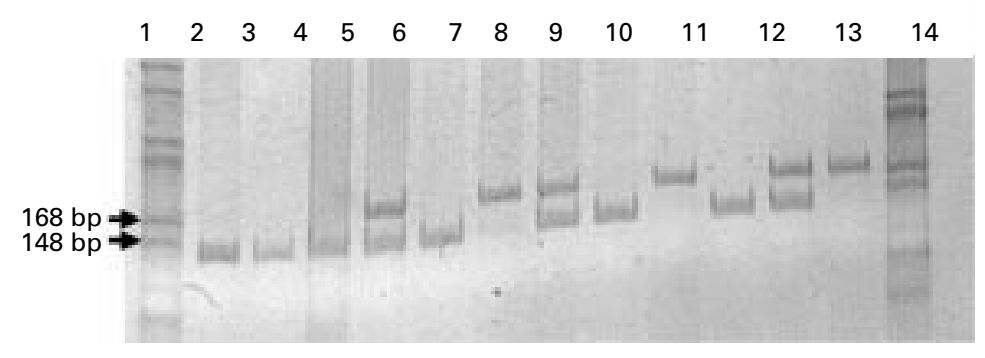

Figure 1 Sample 12\% (wt/vol) acrylamide gel depicting the three $p 53$ codon 72 genotypes detected in the patients with skin cancer and the control population. Lanes 1 and 14 contain molecular weight markers (HaeII digested pBluescript). Lanes 2, 3, 6, 9, and 11 show the single $144 \mathrm{bp}$ band amplified from individuals who are arginine homozygotes for the codon 72 polymorphism. Lanes 7, 10, and 13 show the single $171 \mathrm{bp}$ band amplified from the p53 gene of individuals who are proline homozygotes for the codon 72

polymorphism. Lanes 5, 8, and 12 show both the $144 \mathrm{bp}$ arginine band and the $171 \mathrm{bp}$ proline band amplified from the $p 53$ gene of heterozygous individuals. The gel was stained with ethidium bromide and visualised under UV light.

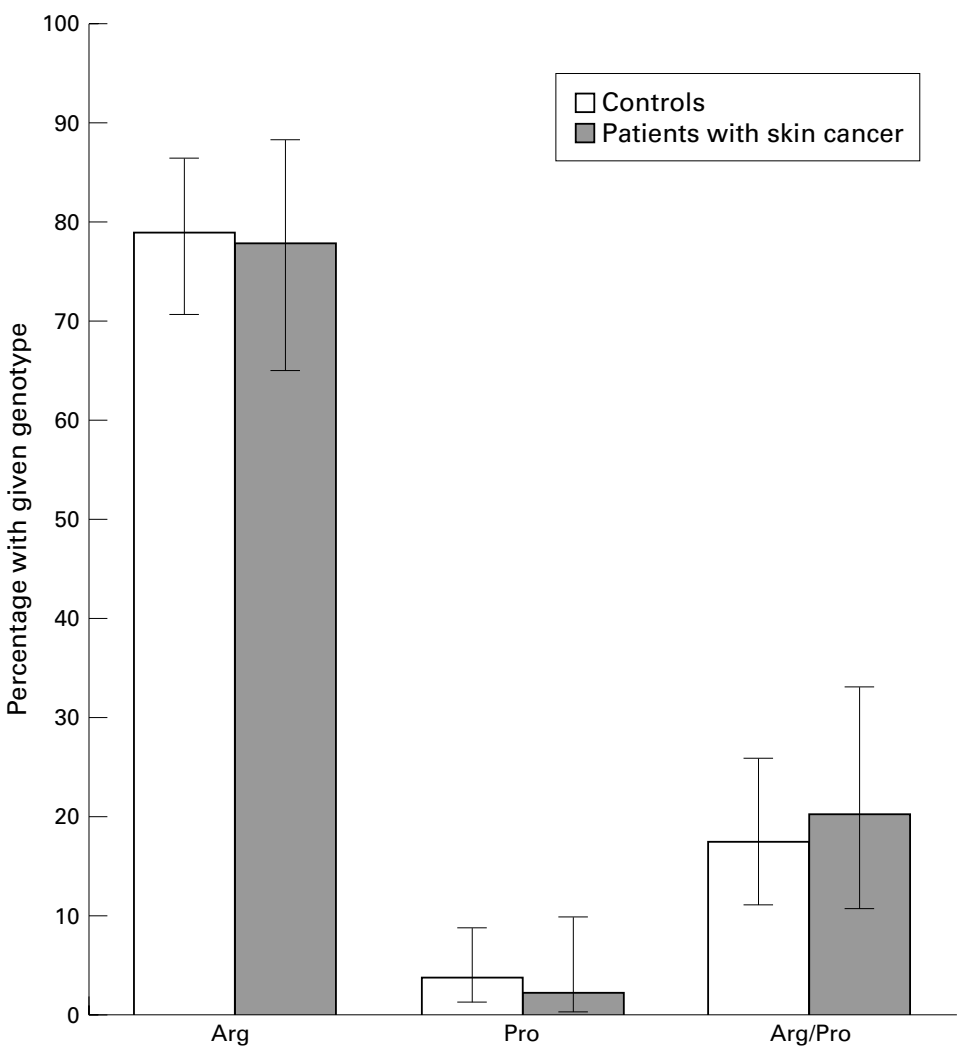

p53 codon 72 genotypes

Figure 2 Bar chart depicting the distribution of $p 53$ codon 72 genotypes in patients with skin cancer and in controls. Error bars represent exact binomial $95 \%$ confidence intervals. Arg, arginine homozygotes; Pro, proline homozygotes; Arg/Pro, heterozygotes.

PCR ANALYSIS OF p53 CODON 72 POLYMORPHISM The amplification of $\mathrm{p} 53$ proline and arginine sequences was performed as previously described by Storey et al. ${ }^{1}$ The products of each of the two polymerase chain reaction (PCR) amplifications were combined and analysed by $12 \%$ (wt/vol) polyacrylamide gel electrophoresis (sample gel shown in fig 1).

STATISTICAL ANALYSIS

Chi squared analysis was used to examine the differences in proportions of the $\mathrm{p} 53$ codon 72 genotypes between the patients with skin cancer and the control group. The odds ratio
(OR) for having the arginine variant and the development of skin cancer was also calculated. Exact binomial 95\% confidence intervals (CI) were calculated for the proportions of the genotypes in each group.

\section{HPV TYPING}

DNA was extracted from viral warts, SCCs, and normal skin by conventional methods. Two PCR strategies were used for the detection of HPV: a degenerate PCR using MY09 and MY11 degenerate primers, as described previously by Manos et $a l,{ }^{9}$ and an $\mathrm{EV}$ specific nested PCR, using CP65-70-EV specific HPV primers, as described previously by Berkhout $e t$ $a l .{ }^{10}$ Using both the degenerate and EV specific primer sets for the detection of HPV has recently been shown to be much more effective than using either in isolation. ${ }^{6}$ All PCR protocols contained positive, negative, and contamination controls, and were performed in a DNA Engine $^{\mathrm{TM}}$ thermal cycler (MJ Research, Massachusetts, USA), initially using Taq polymerase (Promega, Madison, Wisconsin, USA), followed by reamplification with Pfu polymerase (Stratagene, Cambridge, UK). All Pfu generated PCR products were purified by TAE agarose $(2 \% \mathrm{wt} / \mathrm{vol})$ gel electrophoresis and the amplified DNA recovered with the Wizard ${ }^{\mathrm{TM}}$ PCR prep kit (Promega), according to the manufacturer's instructions. All purified fragments were then cloned into pCR Script AMP SK(+) (Stratagene) and cycle sequenced using CY5 labelled M13 universal primer and the Thermosequenase fluorescent labelled primer cycle sequencing kit (Amersham, Little Chalfont, Buckinghamshire, UK). Cycle sequenced products were then analysed on an ALF express genetic analyser (Pharmacia, Uppsalla, Sweden). All sequences generated were aligned with known HPV sequences in the Genome Sequence Database using the Basic Local Alignment Search Tool (BLAST) ${ }^{11}$

\section{Results}

ANALYSIS OF THE DISTRIBUTION OF p53 CODON 72 GENOTYPES AMONG PATIENTS AND CONTROLS

The proportions of p53 codon 72 genotypes found were $78 \%$ arginine homozygous, $2 \%$ proline homozygous, and $20 \%$ heterozygous among patients with skin cancer and $79 \%$ arginine homozygous, $3.5 \%$ proline homozygous, and $17.5 \%$ heterozygous among the control population (fig 2). Statistical analysis showed that there were no significant differences in the distribution of the various alleles between the control group and the skin cancer group $\left(\chi^{2}=0.49\right.$; degrees of freedom $(\mathrm{df})=2$; $\mathrm{p}=0.78)$. The OR for the association of the arginine variant with the development of skin cancer was calculated at 1.06 (95\% CI, 0.49 to $2.3)$.

\section{HPV TYPING}

EV associated HPV types predominate in SCCs (both from renal transplant recipients and immunocompetent patients with skin cancer) and both EV associated HPV types and common cutaneous HPV types are detected in viral warts, again from both skin cancer groups 
Table 1 Human papillomavirus (HPV) types detected in renal transplant recipients (RTRs) and skin lesions from immunocompetent patients (ICPs)

\begin{tabular}{|c|c|c|c|c|}
\hline Patient & Immune status & Lesion & $\begin{array}{l}\text { HPV typing using MY09/MY11 } \\
\text { degenerate primers }\end{array}$ & HPV typing using CP65-70 EV specific primers \\
\hline \multirow[t]{6}{*}{1} & RTR & Wart & HPV-2a/HPV-57 & HPV-23 rel \\
\hline & & NHS & -ve & -ve \\
\hline & & SCC & -ve & HPV-15 \\
\hline & & SCC & $-v e$ & HPV-36/RTRX1 \\
\hline & & SCC & HPV-17/HPV-38-rel & HPV-36 \\
\hline & & SCC & HPV-22 & RTRX1/HPV-38 rel \\
\hline \multirow[t]{3}{*}{2} & RTR & Wart & HPV-57 & HPV-38 rel \\
\hline & & Wart & HPV-10 & HPVx14b \\
\hline & & NHS & -ve & -ve \\
\hline 3 & RTR & Wart & HPV-10 & RTRX5/RTRX6 \\
\hline \multirow[t]{2}{*}{4} & RTR & Wart & HPV-17 & HPV-23-rel \\
\hline & & NHS & -ve & -ve \\
\hline 5 & RTR & Wart & HPV-10 & HPV-2a/HPV-23-rel/RTRX10 \\
\hline \multirow[t]{2}{*}{6} & RTR & Wart & ND & HPV-23 rel \\
\hline & & Wart & ND & RTRX10 \\
\hline 7 & RTR & Wart & -ve & RTRTX10 rel \\
\hline 8 & RTR & Wart & HPV-57 & Unknown HPV type \\
\hline 9 & RTR & Wart & -ve & HPV-23 rel \\
\hline 10 & RTR & SCC & -ve & HPV-38 rel \\
\hline 11 & RTR & SCC & -ve & HPV-38 rel \\
\hline 12 & RTR & SCC & $-\mathrm{ve}$ & -ve \\
\hline 13 & RTR & SCC & - ve & $\mathrm{HPVx} 20$ \\
\hline 14 & RTR & SCC & -ve & HPV-15 \\
\hline 15 & RTR & NHS & $-\mathrm{ve}$ & -ve \\
\hline 16 & RTR & NHS & $-\mathrm{ve}$ & RTRX9/HPV-37/ HPV-15 rel \\
\hline 17 & ICP & Wart & $-\mathrm{ve}$ & HPV-2a/HPV-10 rel \\
\hline \multirow{2}{*}{18} & ICP & Wart & -ve & HPV-20 \\
\hline & & Wart & -ve & HPV-5-rel/HPV-15 rel \\
\hline \multirow[t]{3}{*}{19} & ICP & Wart & -ve & HPV-23 rel \\
\hline & & Wart & -ve & HPV-23 rel \\
\hline & & Wart & -ve & HPV-20/HPV-38 rel \\
\hline 20 & ICP & Wart & -ve & HPVx2b \\
\hline 21 & ICP & Wart & -ve & HPV-23 rel \\
\hline 22 & ICP & SCC & -ve & HPV-20 \\
\hline 23 & ICP & NHS & -ve & HPV-24/HPV-17 \\
\hline \multirow[t]{2}{*}{24} & ICP & SCC & -ve & -ve \\
\hline & & NHS & -ve & -ve \\
\hline 25 & ICP & SCC & -ve & HPV-20 \\
\hline 26 & ICP & SCC & -ve & HPV-21 \\
\hline \multirow[t]{2}{*}{27} & ICP & SCC & -ve & -ve \\
\hline & & NHS & $-\mathrm{ve}$ & HPV-7 \\
\hline \multirow[t]{2}{*}{28} & ICP & SCC & -ve & HPVx2b \\
\hline & & NHS & -ve & -ve \\
\hline 29 & ICP & SCC & -ve & Unknown HPV type \\
\hline 30 & ICP & SCC & -ve & RTRX1 \\
\hline 31 & ICP & SCC & -ve & Unknown HPV type \\
\hline 32 & ICP & SCC & -ve & HPV-23 rel \\
\hline 33 & ICP & SCC & -ve & HPV-23 rel \\
\hline 34 & ICP & SCC & -ve & Unknown HPV type \\
\hline
\end{tabular}

NHS, normal human skin; SCC, squamous cell carcinoma; ND, not done.

(table 1). Normal skin from the patients with skin cancer and the control volunteers was also found to harbour EV associated HPV types; however, the frequency of detection was much lower in the control volunteers than in patients with SCCs or viral warts (table 2). Table 3 summarises our findings.

Table 2 Human papillomavirus (HPV) types detected in the general population

\begin{tabular}{|c|c|c|}
\hline Volunteer & $\begin{array}{l}\text { HPV typing using MY09/MY11 } \\
\text { degenerate PCR primers }\end{array}$ & $\begin{array}{l}\text { HPV typing using CP65-70 "EV } \\
\text { specific HPV primers" }\end{array}$ \\
\hline 1 & -ve & -ve \\
\hline 2 & -ve & -ve \\
\hline 3 & $-\mathrm{ve}$ & -ve \\
\hline 4 & -ve & -ve \\
\hline 5 & -ve & -ve \\
\hline 6 & $-\mathrm{ve}$ & -ve \\
\hline 7 & -ve & -ve \\
\hline 8 & $-\mathrm{ve}$ & HPV-38 rel/unknown HPV \\
\hline 9 & -ve & HPV-23 \\
\hline 10 & -ve & -ve \\
\hline 11 & -ve & $\mathrm{HPV}$ \\
\hline 12 & -ve & -ve \\
\hline 13 & -ve & -ve \\
\hline 14 & -ve & -ve \\
\hline 15 & -ve & -ve \\
\hline 16 & $-\mathrm{ve}$ & -ve \\
\hline 17 & -ve & -ve \\
\hline 18 & -ve & -ve \\
\hline 19 & -ve & -ve \\
\hline 20 & -ve & -ve \\
\hline
\end{tabular}

\section{Discussion}

The p53 codon 72 polymorphism has been implicated as a risk factor in several human cancers. ${ }^{12-14}$ individuals who are proline homozygotes have been shown to have a greater risk of developing lung cancer. ${ }^{15}{ }^{16} \mathrm{~A}$ recent study $^{1}$ suggests that the arginine isoform of p53 facilitates degradation by the E6 protein of low risk HPV-11. Because cutaneous carcinomas frequently harbour multiple low risk HPV types and not high risk HPV-16/18 it would seem that the presence of an arginine instead of a proline could be an important risk factor in the development of these tumours. All of the patients with skin cancer analysed in the study by Storey et al were either arginine homozygotes $(75 \%)$ or heterozygotes $(25 \%),{ }^{1}$ implying that the arginine isoform of p53 confers a susceptibility to tumorigenesis, although only a small number of patients were examined. A second study conducted on 29 immunocompetent patients who had developed skin cancer also suggests that possession of the arginine isoform of p53 is associated with an increased risk of developing HPV associated skin cancer. ${ }^{17}$ 
Table 3 Human papillomavirus (HPV) types detected in patients with skin cancer and in the control population

\begin{tabular}{llll}
\hline Disease status & HPV negative & HPV positive & HPV types detected \\
\hline Controls $(\mathrm{n}=20)$ & $17 / 20$ & $3 / 20$ & EV associated HPV types \\
Normal tissue from cases $(\mathrm{n}=9)$ & $7 / 9$ & $2 / 9$ & EV associated HPV types \\
Warts $(\mathrm{n}=19)$ & $0 / 19$ & $19 / 19$ & EV associated HPV types, common cutaneous HPV types \\
SCCs $(\mathrm{n}=21)$ & $3 / 21$ & $18 / 21$ & EV associated HPV types \\
\hline
\end{tabular}

SCC, squamous cell carcinoma.

Our results do not confirm this hypothesis. We have shown that, at least in a Celtic population, there is no apparent link between the presence of HPV, the p53 codon 72 genotype, and the development of cutaneous carcinomas in both renal transplant recipients and in immunocompetent patients with skin cancer. This conclusion is supported by a recent study that also found no apparent link between a susceptibility to skin cancer and the arginine variant of p53 after renal transplantation. ${ }^{18}$ It is possible, however, that in populations less prone to skin cancer, where the arginine isoform of p53 is less prevalent, ${ }^{19}$ that it could still confer increased susceptibility to malignancy because both our study and that of Marshall and colleagues ${ }^{18}$ were conducted on northern European populations. However, it has been shown that in some populations where the proline isoform predominates, individuals homozygous for the arginine isoform are at no greater risk of developing cervical carcinoma. ${ }^{20-22}$ Whether or not this remains the case for cutaneous malignancies is yet to be established.

The HPV types demonstrated in the patients with skin cancer were also found in the control group, albeit at a much lower frequency. However, EV associated HPV types have been detected in the hair follicles of $46 \%$ of healthy volunteers, ${ }^{23}$ so that a reservoir of EV HPV might exist in a latent or subclinical state in the population as a whole to a greater degree than that found in our study. Recently, intratype variation in the E6 gene of HPV-16 has been suggested as a possible additional factor in determining the susceptibility to cervical cancer associated with the p53 arginine isoform. ${ }^{24}$ HPV types with a $G$ instead of a $T$ at position 350 of the E6 gene were found to be significantly over-represented in arginine homozygous women with cervical cancer.

We conclude that in a Celtic population there is no correlation between the presence of HPV and the development of skin cancer and that $\mathrm{p} 53$ codon 72 polymorphism is not associated with cutaneous malignancies.

We would like to express our sincere thanks to O Tighe, R Ryan, and P Gallagher for providing blood samples from the control population; the medical staff of the dermatology clinic at Beaumont Hospital for providing samples from patients with skin cancer; V Betts and R Conroy for invaluable assistance with the statistical analysis; and C O'Flatharta for technical assistance. We would also like to acknowledge the Irish Cancer Society and the Royal College of Surgeons in Ireland for their financial support.

1 Storey A, Thomas M, Kalita A, et al. Role of a p53 polymorphism in the development of human papillomavirusassociated cancer. Nature 1998;393:229-34.
2 Matlashewski GJ, Tuck S, Pim D, et al. Primary structure polymorphism at amino acid residue 72 of human p53. Mol Cell Biol 1987;7:961-3.

3 Rosenthal AN, Ryan A, Al-Jehani RM, et al. p53 codon 72 polymorphism and risk of cervical cancer in UK. Lancet 1998;352:871-5.

4 Sonoda Y, Saigo PE, Boyd J. p53 codon 72 polymorphism and susceptibility to cervical cancer. $\mathcal{F}$ Natl Cancer Inst 1999;91:557.

5 Zehbe I, Voglino G, Wilander E, et al. Codon 72 polymorphism of $\mathrm{p} 53$ and its association with cervical cancer. Lancet 1999;354:218-19.

6 Surentheran T, Harwood CA, Spink PJ, et al. Detection and typing of human papillomaviruses in mucosal and cutaneous biopsies from immunosuppressed and imunocompetent patients and patients with epidermodysplasia verruciformis: a unified diagnostic approach. I Clin Pathol

De Villiers E-M, Lavergne D, McLaren K, et al. Prevailing papillomavirus types in non-melanoma carcinomas of the
skin in renal allograft recipients. Int $\mathcal{F}$ Cancer 1997;3:35661 .

8 Shamanin V, zur Hausen H, Lavergne D, et al. Human papillomavirus infections in non-melanoma skin cancers from renal transplant recipients and nonimmunosuppressed patients. $\mathcal{F}$ Natl Cancer Inst 1996;88:802-11.

9 Manos MM, Ting Y, Wright DK, et al. Use of polymerase chain reaction amplification for the detection of genital human papillomaviruses. Cancer Cells 1989;7:209-14.

10 Berkhout RJM, Tieben LM, Smitss HL, et al. Nested PCR approach for detection and typing of epidermodysplasia verruciformis-associated human papillomavirus types in cutaneous cancers from renal transplant recipients. F Clin Microbiol 1995;33:690-5.

11 Altschul SF, Gish W, Miller W, et al. Basic local alignment search tool. $f$ Mol Biol 1990;215:403-10.

12 Sorensen DM, Lewark TM, Haney JL, et al. Absence of p53 mutations in squamous carcinomas of the tongue in nonsmoking and nondrinking patients younger than 40 years. Arch Otolaryngol Head Neck Surg 1997;123:503-6.

13 Buller RE, Sood A, Fullenkamp C, et al. The influence of the p 53 codon 72 polymorphism on ovarian carcinogenesis the p53 codon 72 polymorphism on ovarian carcinog

14 Sjalander A, Birgander R, Hallmans G, et al. p53 polymorphisms and haplotypes in breast cancer. Carcinogenesis 1996;17:1313-16.

15 Wang YC, Lee Hs, Chen SK, et al. Prognostic significance of p53 codon 72 polymorphism in lung carcinoma. Eur $\mathcal{F}$ Cancer 1999;5:226-30.

16 Jin X, Roth JA, Amos CI, et al. Higher lung cancer risk for younger African Americans with the Pro/Pro p53 genotype. Carcinogenesis 1995;16:2205-8.

17 Dokianakis DN, Koumantaki E, Billiri K, et al. p53 codon polymorphism as a risk factor in the development of HPVassociated non-melanoma skin cancers in immunocompetent hosts. Int $\mathcal{F}$ Mol Med 2000;5:405-9.

18 Marshall SE, Bordea C, Wojnarowska F, et al. p53 codon 72 polymorphism and susceptibility to skin cancer after renal transplantation. Transplantation 2000;69:994-6.

19 Beckman G, Birgander R, Sjalander A, et al. Is p53 polymorphism maintained by natural selection. Hum Hered 1994;44:266-70.

20 Ngan HY, Liu VW, Liu SS. Risk of cervical cancer is not increased in Chinese carrying homozygous arginine at codon 72 of p53. Br f Cancer 1999;80:1828-9.

21 Yamashita T, Yaginuma Y, Saitoh Y, et al. Codon 72 polymorphism of p53 as a risk factor for patients with human papillomavirus-associated squamous intraepithelial lesions and invasive cancer of the uterine cervix. Carcinogenesis 1999;20:1733-6.

22 Minaguchi T, Kanamori Y, Matsushima M, et al. No evidence of correlation between polymorphism at codon 72 of $\mathrm{p} 53$ and risk of cervical cancer in Japanese patients with human papillomavirus 16/18 infection. Cancer Res 1998; 58:4585-6.

23 Boxman ILA, Berkhout RJM, Mulder LHC, et al. Detection of human papillomavirus DNA in plucked hairs from renal transplant recipients and healthy volunteers. F Invest Dermatol 1997;108:712-15.

24 Van Duin M, Snijders PJ, Vossen MT, et al. Analysis of human papillomavirus type $16 \mathrm{E} 6$ variants in relation to p53 codon 72 polymorphism genotypes in cervical carcinogenesis. f Gen Virol 2000;81:317-25. 